\title{
Photodermatitis and ocular changes in nine horses after ingestion of wild parsnip (pastinaca sativa)
}

\author{
Judith C. Winter ${ }^{1}$, Katharina Thieme ${ }^{1,2^{*}} \mathbb{0}$, J. Corinna Eule ${ }^{2}$, Eva-Maria Saliu ${ }^{3}$, Olivia Kershaw ${ }^{4}$ and \\ Heidrun Gehlen ${ }^{1}$
}

\begin{abstract}
Background: Primary photosensitization rarely occurs in horses and can easily be misinterpreted. Descriptions of the disease in horses after ingestion of parsnip are lacking. The aim of this case series was to describe the dermatological and ocular changes due to photosensitization and to raise awareness of parsnip being a possible aetiologic agent.

Case presentation: Nine horses from three different stables in Berlin and Brandenburg, Germany, presented variable degrees of erythema, scaling, crusting and necrosis of unpigmented skin at the head and prepuce. Horses were of different breeds with a median age of $15 \pm 5.9$ years. A mild leukocytosis was diagnosed in 1/9 horses at admission. Analyzed liver enzymes were within the reference ranges in all horses. Ocular changes were diagnosed as follows: blepharitis (3/9), conjunctivitis (7/9), corneal edema without additional signs of keratitis and/or uveitis (2/9), corneal edema with signs of uveitis (1/9) and photophobia (4/9). One horse developed a fluorescein positive corneal erosion. Skin biopsy (1/9) revealed a moderate to severe acute, eosinophilic and lymphocytic dermatitis with dermal edema and vasculitis. All stables housing these patients fed hay from the same distributer. Analyzed hay samples showed high contents of wild parsnip (plants, seeds, roots). Wild parsnip is widespread in Europe and contains furocoumarins, a family of photodynamic pigments, which may cause primary photodermatitis, keratoconjunctivitis and uveitis. Horses were treated according to severity of clinical symptoms systemically with flunixine meglumine $(1.1 \mathrm{mg} / \mathrm{kg} \mathrm{BW}$ $1-2 x /$ day) or prednisolone (1 mg/kg BW 1x/day). Topically, either gentamicin (3x/day), dexamethasone (2-3x/day) and/ or atropine $(1 \mathrm{x} /$ day) were used. Skin care was provided with almond oil or dexpanthenol (2x/day). All horses were kept in a dark environment or were treated with sunscreen and facemasks. Duration of treatment varied from 6-30 days (median 11.3 days).
\end{abstract}

Conclusion: Ingestion of wild parsnip (Pastinaca sativa) can induce primary photosensitization with dermatitis and ocular injury in horses. In times of extreme weather, hay may alter in botanical composition, resulting in high amounts of uncharacteristic plants causing novel problems.

Keywords: Photosensitization, Parsnip, Furocoumarins, Intoxication, Ocular changes, Cornea, Edema, Erythema, Sunburn, Bergapten

\footnotetext{
*Correspondence: katharina.thieme@fu-berlin.de

1 Equine Clinic: Surgery and Radiology, Freie Universität Berlin, Berlin, Germany

Full list of author information is available at the end of the article
}

\section{Background}

Primary (type I) photosensitization is a clinical condition occurring in animals and humans with accumulation of phototoxic compounds in the skin, cornea and/or mucoid membranes after ingestion of or direct dermal contact with plants or chemicals $[1,2]$. Photosensitization 
outbreaks have been reported from 20 different countries in the peer reviewed literature with the highest number of reports from Australia and Brazil and only 12 reports from Europe [3]. Sheep were the most frequently reported species followed by cattle and horses. Of the reported cases, $68.5 \%$ were suspected or confirmed hepatogenous (type II) photosensitization and only $22.5 \%$ of the cases were diagnosed or suspected to be of the primary type. Different plants have been identified as causal agents, depending on geographic location. St. John's wort (Hypericum perforatum) is common to the United States, South America, Europe, New Zealand, and Australia, Brachiaria decumbens has been identified in Brazil, Colombia and Nigeria. Panicum grasses, Lantana spp. and the fungus Pithomyces chartarum were the most commonly reported etiological agents in Australia [3].

Wild parsnip (Pastinaca sativa) belongs to the Umbelliferae or Apiaceae family that includes celery, parsley and hogweed among others and is widespread in Europe. It prefers direct sunlight and due to the taproot, wild parsnip is draught resistant. Its seeds, flowers and stem contain furocoumarins $[4,5]$. Thirteen different furocoumarins were identified in seeds of wild parsnip. The main compound in mature seeds was bergapten (40.8\% in fresh weight, synonym 5-Metoxypsoralen, 5-MOP). All other identified furocoumarins in wild parsnip are summarized in Table 1 [6]. Since the 1940s, it is known that the plant can cause photodermatitis in humans and was listed as a primary photosensitizing plant with maximum weight of evidence for definite phototoxicity [7-9]. However, reports on photodermatitis in horses and livestock are scarce $[10,11]$. Furocoumarins are the phototoxic agents

Table 1 Furocoumarins identified in immature seeds of wild parsnip (Pastinaca sativa). Information from Kviesis et al., 2019

\begin{tabular}{ll}
\hline Furocoumarin & $\begin{array}{l}\text { Percentage in fresh weight } \\
\text { reported in wild parsnip seeds } \\
\text { (\%) }\end{array}$ \\
\hline Bergapten & 40.8 \\
Byakangelicol & 14.4 \\
Pimpinellin & 10.5 \\
Heraclenin & 8.5 \\
Phellopterin & 7.2 \\
Methoxsalen & 5.7 \\
Isopimpinellin & 4.3 \\
Imperatorin & 3.2 \\
Isobergapten & 2.5 \\
Byakangelicin & 1.3 \\
Heraclenol & 0.5 \\
Psoralen & 0.3 \\
Isobyangelicin & 0.8 \\
\hline
\end{tabular}

in wild parsnip. They are a family of photodynamic pigments produced by a variety of plants. When exposed to UV-A radiation (320-400 $\mathrm{nm}$ ) from sunlight, furocoumarins interact with oxygen and produce ROS (reactive oxygen species). These ROS can damage cell membranes which may lead to edema formation, blistering and epidermal and dermal cell death [4]. To our best knowledge, this case report is the first peer reviewed publication to describe photodermatitis and ocular changes in horses after ingestion of wild parsnip.

\section{Case presentation Stable 1}

The first patient (horse 1) was presented to the Equine Clinic at Freie Universität Berlin in February 2019 with blepharospasm and epiphora non-responding to treatment. It was reported that the horse had experienced episodes of the same clinical symptoms since November 2018. The horse was a 23-year-old Appaloosa-mixed breed stallion with large areas of unpigmented skin at the head and prepuce. Treatment by the referring veterinarian included flunixine meglumine $(1.1 \mathrm{mg} / \mathrm{kg} \mathrm{BW}, 1 \mathrm{x} /$ day, orally) and ofloxacin $(2 \mathrm{x} /$ day, topically). At admission, the horse showed moderate depression, an increased heart ( 56 beats/min), and respiratory rate (26 breaths/min). The unpigmented skin at the head and prepuce showed erythema, thickening, scaling and crusting, especially around the eyes and muzzles (Fig. 1). The horse was reluctant to be touched at the head and showed photophobia. No pathological findings besides severe muco-purulent ocular discharge, blepharitis and conjunctivitis were detected within the ophthalmological examination, which included slit-lamp biomicroscopy (SL-14 ${ }^{\circledR}$, Kowa Company, Sakai, Osaka, Japan), rebound tonometry (TonoVet ${ }^{\circledR}$, icare, Tiolat, Finland), direct ophthalmoscopy (Beta $200^{\circledR}$, Heine, Ettenheim, Germany), and fluorescein staining (I-DEW FLO ${ }^{\circledR}$, ERC Ltd., London, UK). Blood leukocytes (measured in house ${ }^{\mathrm{a}}$ ), GGT, GLDH, AST, Bile acids and ACTH (measured at an external laborator $y^{\mathrm{b}}$ ) were within the reference ranges. The serum selenium- and zinc-concentrations were below the reference ranges (measured at an external laboratory ${ }^{\mathrm{b}}$, selenium: $31.6 \mu \mathrm{g} / \mathrm{L}(100-200 \mu \mathrm{g} / \mathrm{L})$; zinc $6.9 \mu \mathrm{mol} / \mathrm{L}(9.2-19.9 \mu \mathrm{mol} / \mathrm{L}))$. Microbiological examination of the skin revealed growth of Streptococcus dysgalactiae subsp. equisimilis. Skin biopsy showed severe purulent neutrophilic to eosinophilic erosive to ulcerative dermatitis with adhesion of serocellular crusts, epidermal and adnexal hyperplasia and copious amounts of acanthocytes (Fig. 2). A botanical analysis of a hay sample revealed thistle and ribwort besides different grasses. The hay sample was of fair quality 


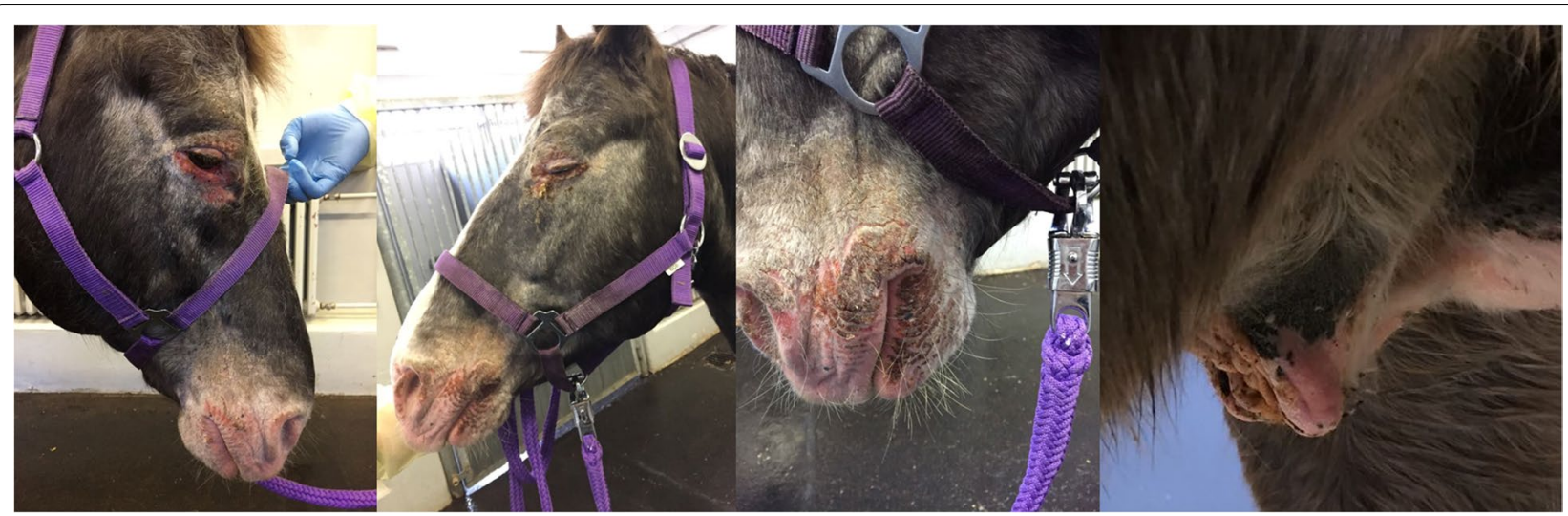

Fig. 1 Horse 1 at admission (day 0) showing erythema, crusting and blistering of the unpigmented skin at the head and prepuce

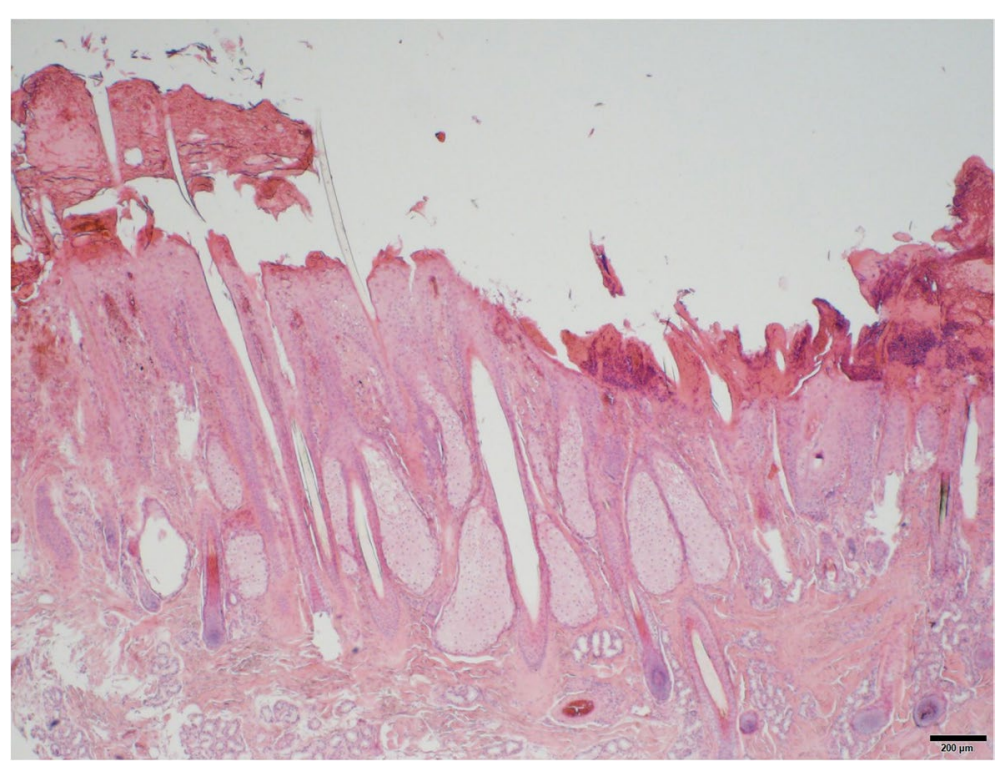

Fig. 2 First skin biopsy of horse 1 with detection of acanthocytes. Hematoxylin and eosin (HE)

with moderate dust concentration. Based on the biopsy results, the suspicion of pemphigus foliaceus was stated. The horse was treated with prednisolone ${ }^{1}$ $(1 \mathrm{mg} / \mathrm{kg} \mathrm{BW}, 1 \mathrm{x} /$ day, orally) and gentamicin eye ointment $^{2}(3 \mathrm{x} /$ day, topically). Skin care was provided with a polyhexanid-solution ${ }^{3}$ and almond oil. The horse was supplemented with selenium ${ }^{4}$ according to manufacturer's instructions. The horse improved significantly throughout the next days and was discharged without clinical symptoms of dermatitis on day 25 after admission. After discharge from the clinic, skin care was provided once daily with almond oil and prednisolone was given in subsequently reduced dosage. Seven days after discharge from the clinic, the horse was presented again with similar symptoms. It was reported that other horses from the same stable showed similar symptoms in unpigmented areas of the skin. Microbiological examination of the skin was repeated and again revealed growth of Streptococcus dysgalactiae subsp. equisimilis while a new skin biopsy showed a moderate to severe acute, eosinophilic to lymphocytic dermatitis with severe superficial dermal edema, vasculitis and multifocal lysis of collagen fibers (Fig. 3). Acanthocytes were not detected. The suspicion of a hypersensitivity reaction type I was stated, as reported in photodermatitis, while the previously suspected diagnosis of pemphigus foliaceus was not confirmed. A new hay sample could not be 


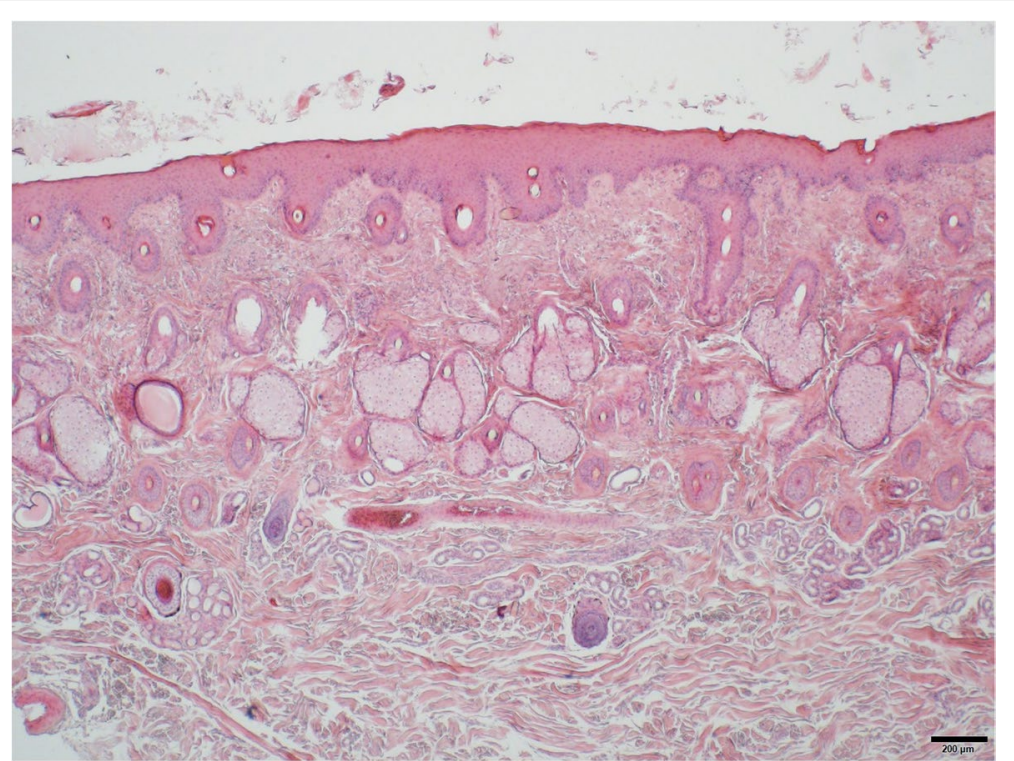

Fig. 3 Second skin biopsy of horse 1. The lesion pattern and the lack of acanthocytes led to the suspected diagnosis of hypersensitivity reaction type I. HE

examined. The horse was treated symptomatically with prednisolone $^{1}(1 \mathrm{mg} / \mathrm{kg} \mathrm{BW}, 1 \mathrm{x} /$ day, orally) as before in combination with azathioprine ${ }^{5}(0.5 \mathrm{mg} / \mathrm{kg} \mathrm{BW}, 1 \mathrm{x} /$ day, orally) and skin care was provided with almond oil. Again, the horse improved rapidly throughout the next days (Fig. 4). The dosage of prednisolone was gradually reduced and ended after 28 days of treatment while azathioprine was continued until day 38 . The horse was discharged at day 68 to a new stable and did not show any symptoms afterwards. While treating this patient, two other horses from a different stable (stable 2) were presented to the clinic with similar symptoms.

\section{Stable 2}

A thoroughbred and a German sportshorse (horse 2 and 3 ), both geldings, 11 and 10 years old, were presented at the clinic in April 2019, with sudden onset of depression, severe photophobia, dermatitis and corneal edema. The symptoms started in both horses about one week after starting a new hay bale, which was not fed to the other horses in this stable. The hay was purchased from the same distributor as in sTable 1. At admission, both horses

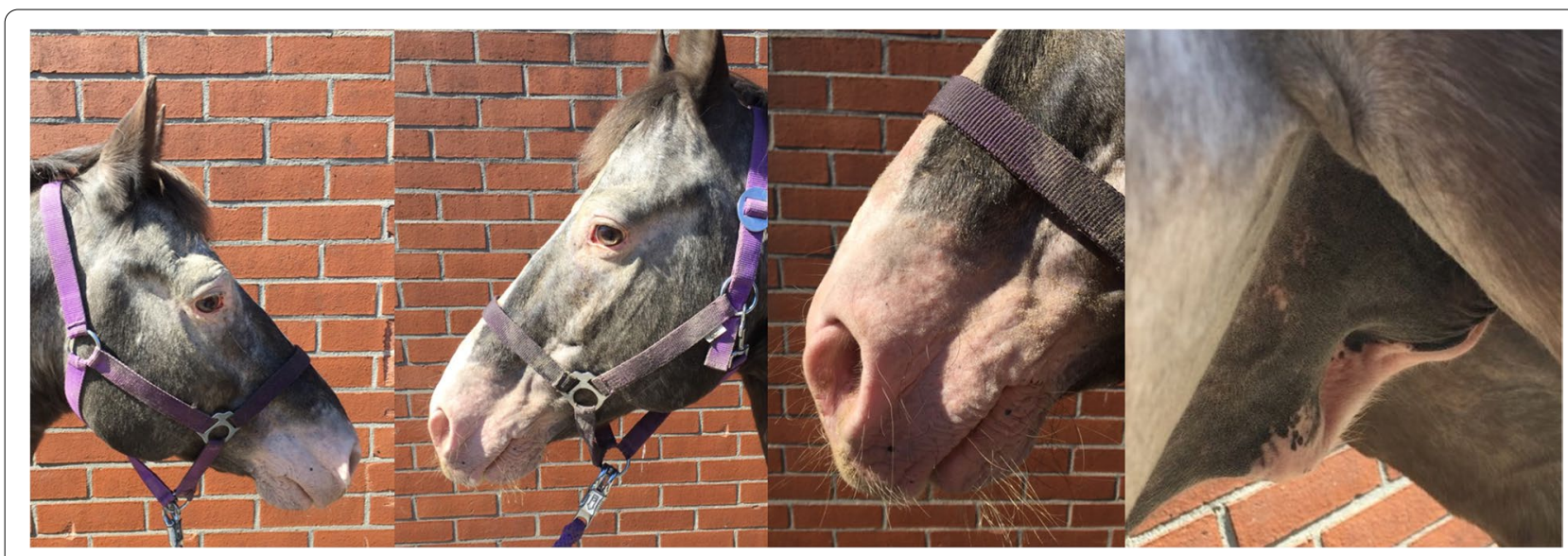

Fig. 4 Horse 1 at day 28 without clinical signs of phytophotodermatitis 
had increased heart and respiratory rates with moderate depression and inappetence. The unpigmented skin at the head and prepuce showed severe crusting and oozing while the pigmented areas did not show alterations (Fig. 5). Both horses had severe blepharospasm, moderate epiphora and severe photophobia. They had a moderate bilateral conjunctivitis, uveitis and generalized corneal edema. Horse 2 had central, fluorescein positive corneal lesions (Fig. 6) while horse 3 showed a unilateral hemorrhage into the anterior chamber (Fig. 7). GGT, GLDH, AP, direct and indirect Bilirubin and bile acids were analyzed in an external laboratory ${ }^{\mathrm{b}}$ and were within the reference ranges in both horses. White blood cell count, total protein and packed cell volume were measured in house ${ }^{\mathrm{a}}$ and were within the reference ranges in both horses. Selenium and zinc were not measured. Botanical analysis of the hay revealed large contents of Pastinaca sativa in the samples. Besides long stems, high amounts of fruits (schizocarps) and a root were identified (Fig. 8). Both patients were treated symptomatically with flunixine meglumine ${ }^{6}(1.1 \mathrm{mg} / \mathrm{kg} \mathrm{BW} 1-2 \mathrm{x} /$ day, orally) and omeprazole ${ }^{7}(2 \mathrm{mg} / \mathrm{kg} \mathrm{BW} 1 \mathrm{x} /$ day, orally), they were stabled and protected from sunlight. Topically, horse 2 received gentamicin ${ }^{2}(3 x /$ day $)$, dexpanthenol

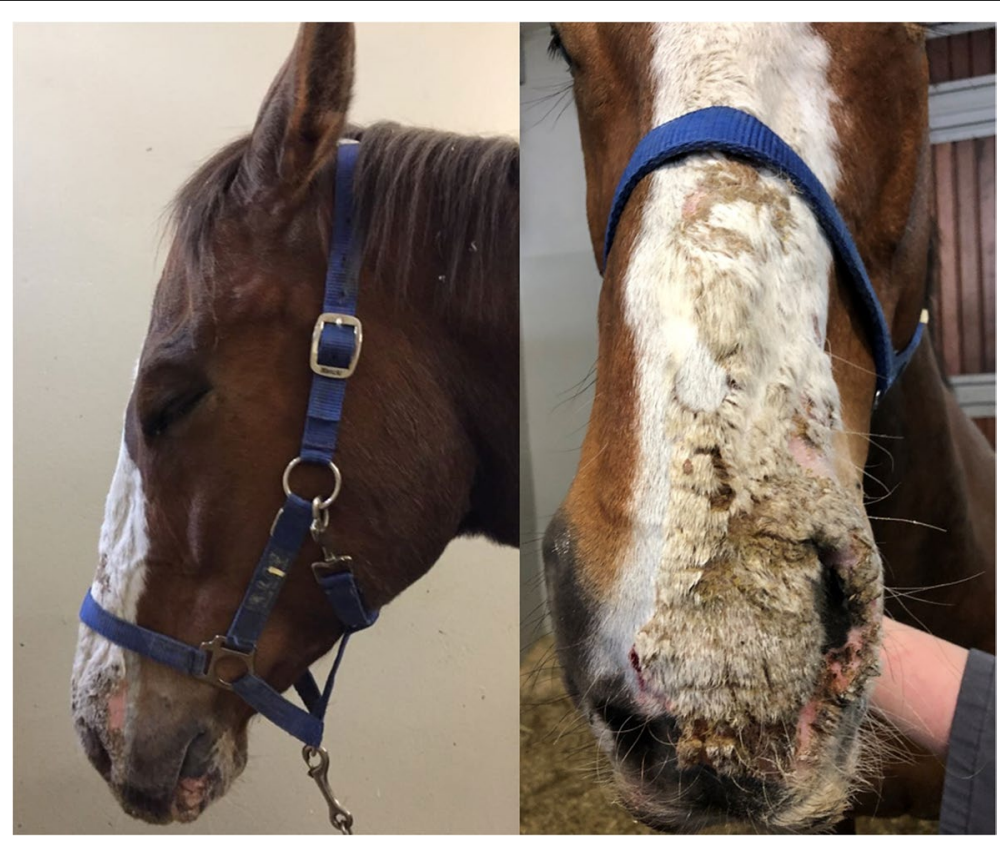

Fig. 5 Horse 3 showing photophobia, depression, and dermatitis at admission (day 0)

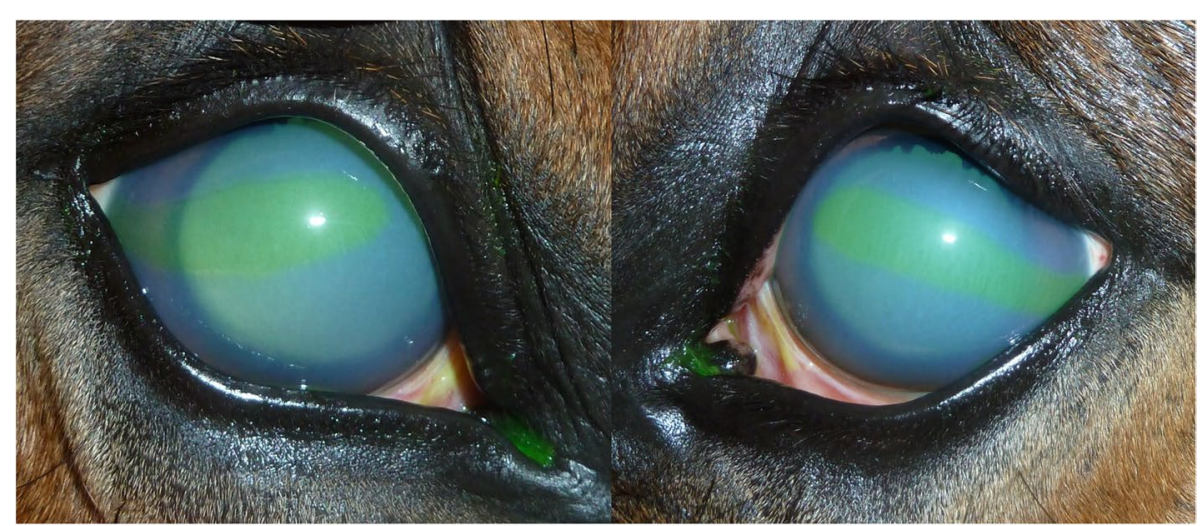

Fig. 6 Horse 2 with marked corneal edema and corneal lesions on day 3. Mydriasis is due to the treatment with atropine eyedrops 


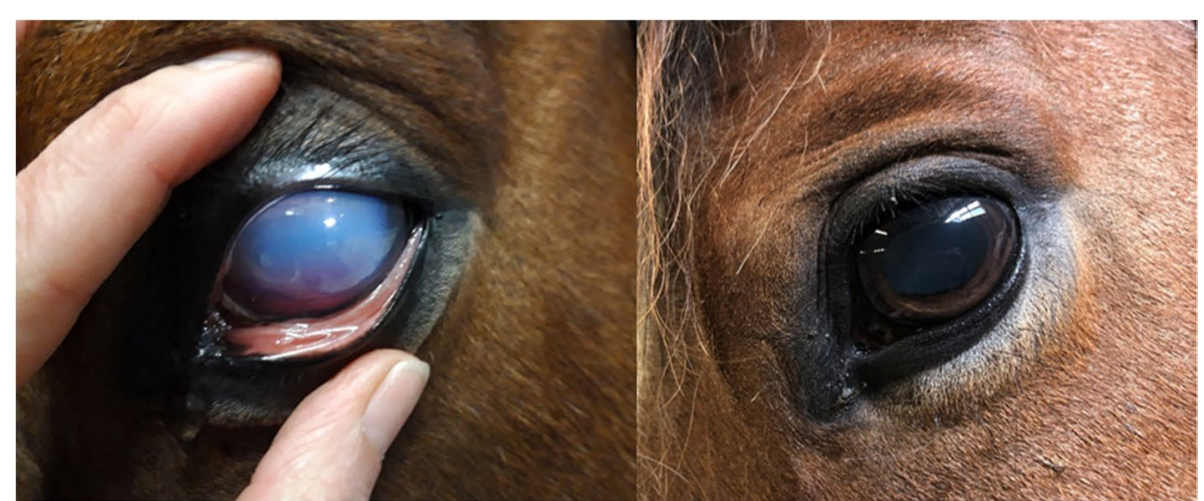

Fig. 7 Horse 3 showing moderate corneal edema at admission (day 0) and mild edema at day 24. There is slight red discoloration in the ventral aspect of the cornea, resulting from hyphema

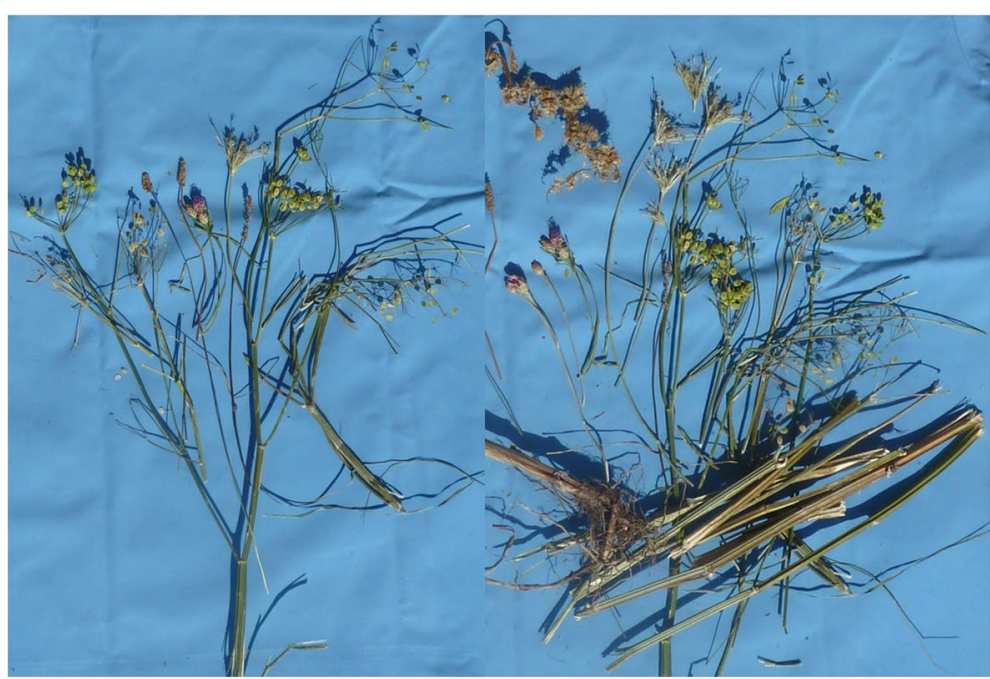

Fig. 8 Parsnip (Pastinaca sativa) found in a hay sample from one of the stables

with retinolpalmitate ${ }^{8}\left(3 x /\right.$ day) and atropine ${ }^{9}$ (1x/day) while horse 3 received gentamicin with dexamethasone ${ }^{10}$ (3x/day), dexpanthenol with retinolpalmitate ${ }^{8}(3 x /$ day), and atropine ${ }^{9}(2 x /$ day). Skin care was provided with dexpathenol ${ }^{12}$. In both horses, ocular changes and dermatitis worsened for two days after admission and then improved significantly throughout the next days. They were discharged after 13 days of treatment. At that time point, horse 2 showed a mild corneal edema in the ventro-temporal areas of both eyes and the following treatment recommendations were made: topical treatment with gentamicin ${ }^{2}$ (3x/day for 3 days), dexpanthenol with retinolpalmitate $^{8}$ (2x/day for one week) and a $\mathrm{NaCl} 5 \%$ solution $^{11}$ ( $3 \mathrm{x} /$ day for one week). Horse $3 \mathrm{did}$ not require further treatment as the cornea did not show any signs of edema and the unilateral hemorrhage into the anterior chamber had resolved. Horses were kept indoors for ten additional days and protected with sunscreen for one month.

\section{Stable 3}

In April 2019, our clinic was contacted to examine six horses in a third stable in the surroundings of Berlin, which suffered from dermatitis and corneal edema. All horses were fed hay from the same distributor as horses $1-3$. Symptoms appeared 3 days after starting a new hay bale. Horses were of different breeds (Hanoverian $(n=1)$; German Sportshorse $(n=1)$, Brandenburg Horse $(n=1)$; Welsh A Pony $(n=1)$, Pony $(n=1)$; Mecklenburg Horse $(n=1))$, two were mares and four were geldings. The horses were 6-30 (median 14 \pm 8.8 ) years old. Botanical analysis of a representative hay sample revealed similar 
findings as described above. Blood leukocytes, GGT and AST (measured in house ${ }^{\mathrm{a}, \mathrm{c}}$ ) were within the reference ranges in all horses. Other blood parameters were not measured. In relation to horses $1-3$, symptoms were mild. Two Horses showed mildly enlarged mandibular lymph nodes, 4/6 horses had mild-moderate blepharospasm, 1/6 had epiphora and 1/6 showed photophobia at the time of examination. Ocular changes were blepharitis $(4 / 6)$, conjunctivitis (4/6), corneal edema (1/6) and miosis or mid-sized pupils despite pretreatment with atropine (5/6). Additional signs of uveitis (e.g., cloudiness of aqueous humor/flare, reduced intraocular pressure) were not observed and topically applied corneal fluorescein retention was negative in all horses. Different degrees of skin alterations (mild-moderate hyperkeratosis, scaling and crusting) were observed in unpigmented areas of the head. Systemic treatment with flunixine meglumine $(1.1 \mathrm{mg} / \mathrm{kg} \mathrm{BW} 1 \mathrm{y} /$ day orally) was necessary in one horse. $5 / 6$ horses received topical ocular treatment with gentamicin ( $3 x /$ day), $3 / 6$ with dexamethasone (2-3x/day) and $5 / 6$ were treated with atropine (1-2x/day). Skin care was provided with dexpanthenol and sunscreen. Medication was provided by the referring veterinarian. Treatment duration was $9 \pm 1.5$ days and all horses showed complete resolution of skin and ocular lesions.

\section{Discussion and conclusions}

In this case series, nine horses from three different stables developed photodermatitis and ocular changes after the ingestion of hay containing high amounts of wild parsnip (Pastinaca sativa). Reports about photosensitization after ingestion of parsnip in horses or other livestock are rare [10, 11]. Nevertheless, photodermatitis and corneal edema after ingestion of furocoumarins have been reported in sheep [12]. When exposed to UV radiation from sunlight, furocoumarins interact with oxygen and produce ROS (reactive oxygen species). These ROS can damage cell membranes in the skin and ocular tissues. Lesions are suspected to occur due to systemic uptake and direct topical contact. The two furocoumarins 5-MOP (=psoralen or bergapten) and 8-MOP are commonly used in PUVA-therapy (psolaren plus UV-A radiation, also called photochemotherapy). This therapy is used to treat skin diseases like vitiligo or psoriasis in humans and has been studied in animal models [13-16]. Barker et al. (1986) reported corneal opacities and, less frequently, lenticular opacities in mice after administration of 5-MOP and 8-MOP. Other studies described chemosis, corneal stromal edema, multiple punctate opacities in the superficial layer of the anterior cortex of the lens, cataract formation, devascularization of the iris, pupil dilation (both in combination with mature cataract), clouding of the anterior chamber and conjunctival hyperemia in the furocoumarin treated animals, while control animals did not show ocular injury $[14,15]$. In the eye, the actions of furocoumarins were limited to the corneal stroma, anterior chamber, iris and anterior lens, which are the anatomic sides of long ultraviolet absorption. The maximum efficiency was found between $320-340 \mathrm{~nm}$ with decreasing efficiency to $330 \mathrm{~nm}$ and no effect for wavelengths longer than $380 \mathrm{~nm}$ was observed. These effects should be differentiated from those of UV-B radiation (280-315 $\mathrm{nm}$ wavelength). At this wavelength, very little light penetrates beyond the cornea and injuries are mostly limited to the corneal epithelium as seen in photokeratitis or "snow blindness" [16, 17]. It was stated that guinea pigs were more susceptible to photosensitizing injury with psoralen than rabbits and mice and experimental data suggest that this might be due to a difference in penetration of 8-MOP into the eye. The threshold dose of 8-MOP required to produce ocular injury in guinea pigs was in the range of $25 \mathrm{mg} / \mathrm{kg}$ body weight while in rabbits a dose of $200 \mathrm{mg} / \mathrm{kg}$ body weight was required [16]. Unfortunately, furocoumarin concentrations were not measured in the present case series due to lack of analytic equipment and invasive nature of sample collection, so it is not possible to compare equine susceptibility to the one in rodents.

In 2018, Quinn et al. developed a grading system for photodermatitis in sheep ranging from Score 0 (no apparent lesions) to 5 (severe lesions) and graded the areas "face and muzzle", "eyes", "ears" and "fleece/body". Scores for the different body areas were added and a total score of $<7$ rated as mild, $<12$ rated as moderate and $\geq 12$ as severe [18]. Unlike in sheep, ears and body were not involved in our cases. This could be due to the fact, that none of the horses had unpigmented ears or other exposed areas of the body. Unpigmented extremities were not involved, probably because they were less exposed to sunlight and did not have direct contact to parsnip. According to the grading system proposed by Quinn et al. the lesions in the areas "face and muzzle" and "eyes" would be categorized as mild in 3/9 horses, moderate in $2 / 9$ horses and severe in $4 / 9$ horses. They would add up to mildly affected animals in $6 / 9$ cases and moderately affected animals in $3 / 9$ cases. We suggest including the presence of photophobia and miosis in the description of the eye lesions (both as mild lesions) an to add an evaluation of the general condition of the animal (not affected or with signs of mild, moderate or severe depression).

In patient 1 , a correct diagnosis could only be stated at the second clinic stay. Possible reasons for the delayed diagnosis might be the large areas of unpigmented skin at the head, which resulted in dermatitis involving almost the complete head. Thus, symptoms appeared similar to Pemphigus foliaceus. In horses, acantholysis can occur 
in Pemphigus foliaceus and secondary, after bacterial infection due to isolation of acanthocytes by bacterial proteases. Both diseases additionally share an unspecific, superficial lymphocytic dermatitis and can easily be confused in the histopathologic examination. Additionally, the analyzed hay sample did not include any plants known to cause photosensitization. This does not exclude the possibility that such plants were present in the hay but may contribute to initial diagnostic difficulties.

The diagnosis of photosensitization in horses 2-9 was made based on the history and clinical picture and was not confirmed pathologically, which is a limitation of the study. Horses 2 and 3 had extensive lesions, yet a relationship to the hay distributor from horse 1 was revealed very quickly. Together with the clinical picture the diagnosis was clear, and we did not see a benefit in a skin biopsy. The skin lesions in horses 4-9 were minor so there was no indication to biopsy.

Dermatitis caused by Streptococcus dysgalactiae subsp. equisimilis, the bacterium cultured from the skin in horse 1 , has been described in children and, in rare cases, in adults [18-21]. The bacterium colonizes many mucosal surfaces of humans and was previously considered nonpathogenic. However, many recent studies revealed that Streptococcus dysgalactiae subsp. equisimilis can cause various soft-tissue infections and occasionally more serious infections including neonatal sepsis, bacteremia, endocarditis, meningitis, peritonitis and arthritis [21, 22]. In horses, Streptococcus dysgalactiae subsp. equisimilis infections have been considered as very infrequent and opportunistic with the highest isolation rate from the reproductive tract. Sampling with nasopharyngeal swabs in healthy horses revealed growth of Streptococcus dysgalactiae subsp. equisimilis in $23.7 \%$ of the cases [19]. Differences in pathogenicity of the bacterium in humans and horses could be due to discrepancies in streptokinase that shows only a $25.4 \%$ identity between both species [20]. Additionally, the type of Streptococcus dysgalactiae subsp. equisimilis and the amount of colony forming units might play a role in expression of clinical symptoms. It is unlikely that Streptococcus equi subsp. equisimilis played a relevant role in the present case.

Wild parsnip is widespread in Europe and the hay distributor reported that it was growing in the field where the relevant hay was harvested, but usually in very low amounts. In the year of 2018, the relation of grasses and wild parsnip changed in favor of parsnip. In Germany and other European countries, the years 2017/2018 were particularly difficult for hay production due to heavy rain in the summer of 2017 and severe draught in 2018. As a result, hay quality was low, prices increased, and hay producers were pressured to use all available grassland for hay production. Hence, weather resistant plants may have gained advantage in growth, leading to higher concentrations of uncharacteristic plants in hay and silage. Horse owners, caretakers and veterinarians are usually confronted with plants from hay samples in their dried condition, without any access to fresh plant material for comparison. This complicates correct identification and may lead to confusions. In dried condition, parsnip might be confused with other plants of the Umbelliferae family such as common hogweed (Heracleum sphodylium), giant hogweed (Heracleum mantegazzianum) or bishop's weed (Ammi majus) all known to cause photosensitization. The giant hogweed can easily be identified by its size, while the fruits of bishop's weed differ significantly from wild parsnip. Other, toxic genera such as hemlock (Conium maculatum), water hemlock (Cicuta) or fool's parsley (Aethusa cynapium) can also be distinguished from wild parsnip by the shape of their fruits. Hogweed fruits on the opposite look very similar to wild parsnip. When fresh, both plants can easily be distinguished by the color of their blossoms: parsnip blooms yellow while, hogweed produces white blossoms [12, 23]. As Heracleum sphodylium also contains furocoumarins and is known to cause photodermatitis in humans, horses and other animals, the final classification might not be necessary for adequate treatment of the patient. Animals ingesting plants containing furocoumarins frequently develop ocular alterations. This is not observed in animals showing symptoms of photosensitivity after ingesting other types of plants such as St. John's wort. Thus, ocular changes combined with symptoms of photosensitivity may be the cardinal symptom for intoxications with furocoumarins.

\section{Abbreviations}

ACTH: Adrenocorticotropic hormone; AST: Aspartate-aminotransferase; BW: Body weight; GGT: Gamma-glutamyltransferase; GLDH: Glutamatedehydrogenase; subsp: Subspecies.

\section{Acknowledgements}

These data were presented as a poster presentation at the 2019 Congress of the European College of Equine Internal Medicine in Valencia, the 2019 Congress of the European Society of Veterinary and Comparative Nutrition in Turin and as an oral presentation at the 2021 Congress of the European College of Veterinary Ophthalmology in an online format.

\section{Authors' contributions}

$K T, J W, J C E$ and $H G$ were equally involved in the clinical assessment of the horses and EMS in the analysis of the hay samples. The manuscript was prepared by JW and KT. The pathological examination was performed by OK. All authors have read and approved the final manuscript (KT, JW, JCE, EMS, HG, OK)

\section{Funding}

Open Access funding enabled and organized by Projekt DEAL.

Availability of data and materials

All data generated or analyzed during this study will be available from the corresponding author on reasonable request. 


\section{Declarations}

Ethics approval and consent to participate

There were no associated ethical issues in this clinical study and all the owners gave their consent for all tests and procedures.

\section{Consent for publication}

All horse owners gave their consent, that their horses' data and photographs could be used for scientific purposes.

\section{Competing interests}

The authors declare no conflict of interest.

\section{Author details}

'Equine Clinic: Surgery and Radiology, Freie Universität Berlin, Berlin, Germany. ${ }^{2}$ Small Animal Clinic, Freie Universität Berlin, Berlin, Germany. ${ }^{3}$ Institute of Animal Nutrition, Freie Universität Berlin, Berlin, Germany. ${ }^{4}$ Institute of Pathology, Freie Universität Berlin, Berlin, Germany.

\section{Manufacturer's addresses}

${ }^{a}$ VetScan HM 5, Scil animal care company GmbH, Viernheim, Germany bLaboklin GmbH \& Co. KG, Bad Kissingen, Germany; certified according to DIN EN ISO/IEC 17025:2005

${ }^{C}$ Reflotron ${ }^{\circledR}$ plus Roche Deutschland Holding GmbH, Grenzach-Wyhlen/ Germany

${ }^{1}$ Equisolon, Böhringer Ingelheim Vetmedica GmbH, Ingelheim, Germany ${ }^{2}$ Gent-Ophtal ${ }^{\circledR}$, Ursapharm Arzneimittel GmbH, Saarbrücken, Germany ${ }^{3}$ ProntoVet ${ }^{\circledR}$, B.Braun Melsungen AG, Melsungen, Germany

${ }^{4}$ Navalis Orthosa ${ }^{\circledR}$ Selen, Navalis ${ }^{\circledR}$ nutraceuticals $\mathrm{GmbH}$, Filderstadt

${ }^{5}$ Azathioprin HEXAL ${ }^{\circledR} 100 \mathrm{mg}$, Hexal AG, Holzkirchen, Germany

${ }^{6}$ Flunido ${ }^{\circledR} 5 \%$, cp-pharma Handelsgesellschaft mbH, Burgdorf, Germany ${ }^{7}$ Equizol $^{\circledR}, \mathrm{cp}$-pharma Handelsgesellschaft mbH, Burgdorf, Germany ${ }^{8}$ Vitamycin ${ }^{\circledR}$ Augensalbe, cp-pharma Handelsgesellschaft mbH, Burgdorf, Germany

${ }^{9}$ Atropin-POS 0,5 \%, Ursapharm Arzneimittel GmbH, Saarbrücken, Germany

${ }^{10}$ Dexa-Gentamicin, Ursapharm Arzneimittel GmbH, Saarbrücken, Germany

${ }^{11}$ Omni-Sorb ${ }^{\circledR}$, Omnivision GmbH, Wien, Austria

${ }^{12}$ Panthenol-ratiopharm ${ }^{\circledR}$ Wundbalsam, ratiopharm GmbH, Ulm, Germany

Received: 19 October 2021 Accepted: 26 January 2022

Published online: 26 February 2022

\section{References}

1. Quinn JC, Kessell A, Weston LA. Secondary plant products causing photosensitization in grazing herbivores: their structure, activity and regulation. Int J Mol Sci. 2014;15(1):1441-65.

2. Barrington GM. Integumentary System In: Kahn CM, editor. The Merck Veterinary Manual. 10. London, United Kingdom Wiley; 2010. p. 665 - 800

3. Chen Y, Quinn JC, Weston LA, Loukopoulos P. The aetiology, prevalence and morbidity of outbreaks of photosensitisation in livestock: A review. PLoS ONE. 2019;14(2):e0211625.

4. Hankinson A, Lloyd B, Alweis R. Lime-induced phytophotodermatitis. J Community Hosp Intern Med Perspect. 2014;4(4):25090.

5. Roth L, Daunderer M, Kormann K. Giftpflanzen-Pflanzengifte. 4th ed. Hamburg: Nikol Verlagsgesellschaft mbH \& Co KG; 1994.

6. Kviesis J, Klimenkovs I, Arbidans L, Podjava A, Klavins M, Liepins E. Evaluation of furanocoumarins from seeds of the wild parsnip (Pastinaca sativa L. s.I.). J Chromatogr B Analyt Technol Biomed Life Sci. 2019;1105:54-66.

7. Bellringer H. Phyto-photo-dermatitis BMJ. 1949;1 (4613):984.

8. Lutchman L, Inyang V, Hodgkinson D. Phytophotodermatitis associated with parsnip picking. Emerg Med J. 1999;16(6):453-4

9. Walling AL, Walling HW. Phytophotodermatitis induced by wild parsnip. Dermatol Online J. 2018;24(2):13030.

10. Martinson KB, Hovda L, Murphy M, Weicherding P. Plants poisonous or harmful to horses in the North Central United States: University of Minnesota Cooperative Extension Service; 2007

11. Stegelmeier BL, Colegate SM, Knoppel EL, Rood KA, Collett MG. Wild parsnip (Pastinaca sativa)-induced photosensitization. Toxicon. 2019;167:60-6.
12. Mauldin EA, Peters-Kennedy J. Integumentary system. Jubb, Kennedy \& Palmer's Pathology of Domestic Animals: Volume 1. 2016:509

13. Barker FM, Dayhaw-Barker P, Forbes PD, Davies RE. Ocular effects of treatment with various psoralen derivatives and ultraviolet-A (UVA) radiation in HRA/Skh hairless mice. Acta Ophthalmol. 1986;64(4):471-8.

14. Cloud TM, Hakim R, Griffin AC. Photosensitization of the Eye with Methoxsalen: I. Acute Effects Arch Ophthalmol. 1960;64(3):346-51.

15. Cloud TM, Hakim R, Griffin AC. Photosensitization of the Eye with Methoxsalen: II. Chronic Effects Arch Ophthalmol. 1961;66(5):689-94.

16. Freeman RG, Troll D. Photosensitization of the eye by 8-methoxypsoralen. J Invest Dermatol. 1969;53(6):449-53.

17. Delic NC, Lyons JG, Di Girolamo N, Halliday GM. Damaging Effects of Ultraviolet Radiation on the Cornea. Photochem Photobiol. 2017:93(4):920-9.

18. Abdolrasouli A, Hemmati Y, Amin A, Roushan A, Butler I. Lancefield group $G$ Streptococcus dysgalactiae subsp. equisimilis: an unusual aetiology of perianal streptococcal dermatitis acquired from heterosexual oral-anal intercourse. Int J STD AIDS. 2012;23(12):911-2.

19. Acke E, Midwinter A, Lawrence K, Gordon S, Moore S, Rasiah I, et al. Prevalence of Streptococcus dysgalactiae subsp. equisimilis and S. equi subsp. zooepidemicus in a sample of healthy dogs, cats and horses. N Z Vet J. 2015;63(5):265-71.

20. Preziuso S, Pinho M, Attili A, Melo-Cristino J, Acke E, Midwinter A, et al. PCR based differentiation between Streptococcus dysgalactiae subsp. equisimilis strains isolated from humans and horses. Comp Immunol Microbiol Infect Dis. 2014;37(3):169-72.

21. Yamaguchi T, Kawahara R, Katsukawa C, Kanki M, Harada T, Yonogi S, et al. Foodborne outbreak of group $\mathrm{G}$ streptococcal pharyngitis in a school dormitory in Osaka. Japan J Clin Microbiol. 2018:56(5):e01884-e1917.

22. Quinn JC, Chen Y, Hackney B, Tufail MS, Weston LA, Loukopoulos P. Acuteonset high-morbidity primary photosensitisation in sheep associated with consumption of the Casbah and Mauro cultivars of the pasture legume Biserrula. BMC Vet Res. 2018;14(1):11.

23. Ivens P. Hogweed suspected of causing primary photosensitisation in a horse. Vet Rec. 2011;169(3):81

\section{Publisher's Note}

Springer Nature remains neutral with regard to jurisdictional claims in published maps and institutional affiliations.

Ready to submit your research? Choose BMC and benefit from

- fast, convenient online submission

- thorough peer review by experienced researchers in your field

- rapid publication on acceptance

- support for research data, including large and complex data types

- gold Open Access which fosters wider collaboration and increased citations

- maximum visibility for your research: over 100M website views per year

At BMC, research is always in progress.

Learn more biomedcentral.com/submissions 